\title{
PRINCIPAL COMPONENT ANALYSIS (PCA) EVALUATION OF LIQUID CHROMATOGRAPHY- MASS SPECTROMETRY (LC-MS) DATASETS OF Ganoderma boninense INTRACELLULAR METABOLITES
}

\section{ZAIN NURAZAH*; ABRIZAH OTHMAN* and UMI SALAMAH RAMLI*}

\begin{abstract}
Liquid chromatography-mass spectrometry (LC-MS) has become a powerful analytical technique for studying broad coverage of chemical datasets describing complex biological systems and events. In order to interpret the underlying information in such datasets, multivariate analysis method such as principal component analysis (PCA) is crucial for multiple sample comparisons and multivariate data reduction. $P C A$ has been used for evaluation of large-scale datasets derived from LC-MS analysis of fungal metabolites for many applications. Therefore, in this study, we describe on PCA as a descriptive tool to cope with large LC-MS datasets of intracellular metabolites of oil palm basal stem rot (BSR) fungal pathogen, Ganoderma boninense from in vitro liquid culture system. The results revealed a classification and grouping of $\mathrm{G}$. boninense intracellular metabolites according to time trend, where the primary metabolites, i.e. glucose, gluconic acid, mannitol and malic acid were found differentially expressed in $\mathrm{G}$. boninense. The presented findings suggest that the PCA model provides a general approach for handling, analysis and interpretation of large LC-MS datasets to reveal time-dependent changes of intracellular metabolites that may indicate G. boninense developmental process in vitro.
\end{abstract}

\section{Keywords: PCA, multivariate analysis, liquid chromatography-mass spectrometry, Ganoderma.}

Received: 2 June 2020; Accepted: 14 September 2020; Published online: 20 November 2020

\section{INTRODUCTION}

A range of technical approaches have been used for the analysis of metabolites in biological samples including liquid chromatographymass spectrometry (LC-MS). The technological advancement of LC-MS has been extensively reviewed elsewhere (Hayden et al., 2019; Yuan et al., 2019; Robison et al., 2018) and increasingly become leading-edge instrumental developments due to its high sensitivity for the detection of metabolic

Malaysian Palm Oil Board,

6 Persiaran Institusi, Bandar Baru Bangi,

43000 Kajang, Selangor, Malaysia.

E-mail: nurazah@mpob.gov.my signatures in the analysis of pathogen (Chen et al., 2019; Tugizimana et al., 2019). LC-MS analysis generates information-rich multivariate datasets comprising of samples and variables that provide insight into the relationship of metabolites and changes in the status of complex biological systems and events. To interpret the underlying information within the complex LC-MS datasets towards reliable conclusions, it is of great importance that a practical and organised statistical modelling tool is applied.

The multivariate statistical analysis and data mining approaches for pattern discovery have facilitated information retrieval from the vast amount of biological data (Ivosev et al., 2008; Jonsson et al., 2005; Holmes and Antti, 2002). Principal component analysis (PCA) is a popular dimension 
reduction method to reduce high-dimensional data into a low-dimensional subspace component (DesRochers et al., 2020; Gotthardt et al., 2019; de Falco et al., 2019; Diana and Tommasi, 2002). The information needs to be interrogated in a way that enables scientists to make a prediction and increases the interpretability of the large datasets, at the same time, minimising the loss of important information. Furthermore, PCA provides knowledge on the nature of the variables and assigns a large number of variables to a smaller number of groups that can be more readily visualised and understood (Ivosev et al., 2008).

Ganoderma boninense is a well-recognised basidiomycete fungal pathogen associated with oil palm basal stem rot disease (Chong et al., 2017) that causes significant economic losses by reducing the yield of fresh fruit bunches. The ability to generate and mine large-scale datasets of biological molecules, i.e. proteins, metabolites combined with recent advances in high-throughput instrumentation analysis and data mining tools may have a profound effect on the systems-level understanding of the pathogenicity of G. boninense (Othman et al., 2019). Multivariate statistical analysis and data mining such as PCA has been used to evaluate large-scale datasets derived from LC-MS analysis of fungal metabolites for many applications, i.e. differentiation of virulent and avirulent species, assessment of metabolic behaviour in different culture environments and evaluation of metabolic difference in spores and mycelia (DesRochers et al., 2020; Siless et al., 2018; Son et al., 2018; Liu et al., 2015). Harnessing the complexity of the metabolomics data derived from the various analytical platforms remains as major challenges in metabolomics. To the best of our knowledge, up to now, there are no studies reported on the statistical evaluation and data mining of large-scale datasets from the G. boninense pathogen.

In that perspective, our study aimed to focus on multivariate statistical evaluation using PCA to cope with the large-scale LC-MS datasets from the G. boninense intracellular metabolites. This approach is important for a robust and reliable comparison of G. boninense intracellular metabolites measured over time using statistical strategy applied to the complex LC-MS datasets.

\section{MATERIALS AND METHODS}

\section{Chemicals and Reagents}

Acetonitrile, methanol and acetic acid of high performance liquid chromatography (HPLC) grade were purchased from Merck, Germany. Difco $^{\mathrm{TM}}$ potato dextrose agar (PDA) and Difco ${ }^{\mathrm{TM}}$ malt extract broth (MEB) were purchased from Becton, Dickinson and Company (BD), USA. Ammonium phosphate monobasic $\left[\left(\mathrm{NH}_{4}\right) \mathrm{H}_{2} \mathrm{PO}_{4}\right]$, ammonium phosphate dibasic $\left[\left(\mathrm{NH}_{4}\right)_{2} \mathrm{HPO}_{4}\right]$, potassium phosphate monobasic $\left(\mathrm{KH}_{2} \mathrm{PO}_{4}\right)$, magnesium sulphate heptahydrate $\mathrm{MgSO}_{4} \cdot\left(7 \mathrm{H}_{2} \mathrm{O}\right)$, iron (III) sulphate heptahydrate $\left(\mathrm{FeSO}_{4} \cdot 7 \mathrm{H}_{2} \mathrm{O}\right)$, zinc sulphate heptahydrate $\left(\mathrm{ZnSO}_{4} \cdot 7 \mathrm{H}_{2} \mathrm{O}\right)$, sodium molybdate dihydrate $\left(\mathrm{NaMoO}_{4} \cdot 2 \mathrm{H}_{2} \mathrm{O}\right)$, copper (II) sulphate pentahydrate $\left(\mathrm{CuSO}_{4} \cdot 5 \mathrm{H}_{2} \mathrm{O}\right)$, manganese (II) chloride tetrahydrate $\left(\mathrm{MnCl}_{2} \cdot 4 \mathrm{H}_{2} \mathrm{O}\right)$, 2-N-morpholinoethanesulphonic acid (MES) and glucose $(\geq 99.5 \%)$ were purchased from SigmaAldrich, USA. Water was purified by a Milli-Q system (Millipore, USA).

\section{Fungal Material Preparation and Liquid Culture Condition}

Ganoderma boninense isolate PER71 was obtained from the Plant Pathology and Biosecurity Unit, Malaysian Palm Oil Board, Bangi, Selangor, Malaysia. G. boninense liquid culture protocol was previously described by Rees (2006). G. boninense was grown on Difco ${ }^{\mathrm{TM}}$ potato dextrose agar (PDA) (Becton, Dickinson and Company, USA) plate and incubated at $27 \pm 1^{\circ} \mathrm{C}$ for eight days. A plug of 9.5 $\mathrm{mm}$ of $\mathrm{G}$. boninense was harvested from eight days old culture on PDA plate. Biological replicates for each $G$. boninense were grown by inoculating one plug from each six individual PDA plates into $50 \mathrm{ml}$ of malt extract broth (MEB) (Becton, Dickinson and Company, USA) in $175 \mathrm{~cm}^{3}$ Nunclon ${ }^{\mathrm{TM}}$ cell culture flask (ThermoFisher Scientific, USA). A total of six mycelial plugs were grown in the cell culture flask at $27 \pm 1^{\circ} \mathrm{C}$ for six days. After six days, the G. boninense mycelial plugs were then collected on sterile filter paper, washed three times with sterile distilled water and inoculated into the $50 \mathrm{ml}$ of growth medium supplemented with carbon and nitrogen sources in $175 \mathrm{~cm}^{3}$ Nunclon $^{\mathrm{TM}}$ cell culture flask (ThermoFisher Scientific, USA). The growth medium consisted of: $0.9 \mathrm{~g}\left(\mathrm{NH}_{4}\right) \mathrm{H}_{2} \mathrm{PO}_{4} ; 2.0 \mathrm{~g}\left(\mathrm{NH}_{4}\right)_{2} \mathrm{HPO}_{4} ; 1.0 \mathrm{~g}$ $\mathrm{KH}_{2} \mathrm{PO}_{4} ; 0.5 \mathrm{~g} \mathrm{MgSO}_{4} .\left(7 \mathrm{H}_{2} \mathrm{O}\right) ; 1 \mathrm{ml}$ of $100 x$ trace elements $\left(1.11 \mathrm{~g} \mathrm{FeSO}_{4} .7 \mathrm{H}_{2} \mathrm{O}, 5.75 \mathrm{~g} \mathrm{ZnSO}_{4} .7 \mathrm{H}_{2} \mathrm{O}\right.$, $0.09 \mathrm{~g} \mathrm{NaMoO}_{4} .2 \mathrm{H}_{2} \mathrm{O}, 0.1 \mathrm{~g} \mathrm{CuSO}_{4} .5 \mathrm{H}_{2} \mathrm{O}$ and 0.21 g $\mathrm{MnCl}_{2} .4 \mathrm{H}_{2} \mathrm{O}$ in $200 \mathrm{ml}$ distilled water); $3.9 \mathrm{~g}$ 2-N-morpholinoethanesulphonic acid (MES) $\mathrm{pH}$ $5.5 ; 5.0 \mathrm{~g}$ glucose in 1 litre distilled water. The culture was grown at $27 \pm 1^{\circ} \mathrm{C}$. The G. boninense intracellular metabolites were sampled over time, on Days 2, 4, 6 and 8 for analysis.

\section{Metabolite Extraction}

The G. boninense mycelia containing intracellular metabolites were freeze-dried for two days using a FreeZone ${ }^{\circledR}$ Freeze Drier System (Labconco, USA). 
About $0.1 \mathrm{~g}$ of the powdered mycelia was dissolved in $5 \mathrm{ml}$ of methanol (Merck, Germany), vortexed for $1 \mathrm{~min}$ and sonicated for $15 \mathrm{~min}$. The mixture was then centrifuged at $89 \times \mathrm{g}$ for $15 \mathrm{~min}$ at $25^{\circ} \mathrm{C}$. The supernatant was collected and the pellet was reextracted with $5 \mathrm{ml}$ of methanol, vortexed for $1 \mathrm{~min}$, sonicated for $15 \mathrm{~min}$ and then centrifuged at $89 \mathrm{xg}$ for $15 \mathrm{~min}$ at $25^{\circ} \mathrm{C}$. The supernatant was collected and pooled with the previous supernatant and dried under a nitrogen stream. The extract was dissolved in $3 \mathrm{ml}$ of methanol (Merck, Germany) and filtered through a $0.2 \mu \mathrm{m}$ cellulose acetate Minisart syringe filter (Merck, Germany). The filtered extracts were stored at $-20^{\circ} \mathrm{C}$ before analysis. An aliquot of $10 \mu \mathrm{l}$ was subjected to liquid chromatography-mass spectrometry (LC-MS) analysis.

\section{Liquid Chromatography-Mass Spectrometry (LC- MS)}

Ganoderma boninense intracellular metabolites were separated using the Ultimate 3000 High Performance Liquid Chromatography (HPLC) (Thermo Scientific, USA), where the separation was achieved on a Reversed-Phase Acclaim $^{\mathrm{TM}}$ Polar Advantage II (C18 $4.6 \times 250$ mm length, $5 \mu \mathrm{m}$ particle size) (Thermo Scientific, USA). With a gradient elution program at a flow rate of $1.0 \mathrm{ml} \mathrm{min} \mathrm{m}^{-1}$ and an injection volume of $5 \mu \mathrm{l}$, the chromatographic separation was performed at $37^{\circ} \mathrm{C}$. The mobile phase A was an aqueous solution containing $0.1 \%$ (v/v) acetic acid (Merck, Germany) and mobile phase B was acetonitrile (Merck, Germany) containing $0.1 \%$ (v/v) acetic acid (Merck, Germany). The gradient program was started at $5 \%$ of B and increased linearly to $60 \%$ of B for $22 \mathrm{~min}$. To minimise the carry-over between injections, the column was washed for $5 \mathrm{~min}$ and equilibrated for 2 min. Blank water injection was applied in-between the sample injection. All samples and replicates were injected simultaneously as one batch in random order to distinguish between technical and biological variations.

For the MS analysis, the HPLC system was coupled to a MicrOTOF- ${ }^{\mathrm{TM}}$ quadrupole-time-offlight (QTOF) mass spectrometer (Bruker Daltonik, Germany) with an electrospray ionisation (ESI) interface operating in negative ion mode and controlled by the HyStar Application version 3.2 software (Bruker Daltonik, Germany). The column effluent was set at $1.0 \mathrm{ml} \mathrm{min}{ }^{-1}$. A split ratio of $1: 4$ was used, so the flow arriving at the detector was $250 \mu \mathrm{min}^{-1}$. Nitrogen was used as nebulising gas at 4.1 bar and 9.0 litre $\mathrm{min}^{-1}$ flow rate. The temperature and voltage of the capillary were set at $200^{\circ} \mathrm{C}$ and $+3.5 \mathrm{kV}$, respectively. The full MS scan covered the mass range of $50-1000 \mathrm{~m} / \mathrm{z}$. Tandem mass spectrometry (MS/MS) parameters were set to improve mass fragmentation, with collision energy varying from $15-35 \mathrm{eV}$.

\section{LC-MS Data Evaluation and Principal Component Analysis (PCA)}

LC-MS data evaluation was performed with DataAnalysis version 4.2 for chromatogram analysis and ProfileAnalysis version 2.1 (Bruker Daltonik, Germany) for PCA. Compounds in the raw LC-MS data were extracted using MS algorithm version 3.0, sensitivity 99\%, absolute intensity of 1000 and minimum peak valley of $10 \%$ under Find Compounds - Chromatogram module in DataAnalysis version 4.2, for comprehensive detection of all compounds in LC-MS run. PCA was performed based on bucket tables of the MS data. The data was integrated from 2.0-22 $\mathrm{min}$ and $50.5-999.5 \mathrm{~m} / \mathrm{z}$ in time- and $\mathrm{m} / \mathrm{z}$-buckets using time alignment parameters and spectral background subtraction in the advanced bucketing approach. The multivariate statistics, PCA, was performed where data were Pareto scaled. The LC-MS data were then normalised to the sum of bucket values for an analysis, which considered the total intensity in analysis and suitable for unknown samples. A full cross and test set validations were applied to validate the PCA model. In ProfileAnalysis version 2.1 (Bruker Daltonik, Germany), the SmartFormula module was used to process the accurate mass data of molecular ions. The list of possible elemental formulas that use $\mathrm{CHNO}$ algorithm such as minimum/maximum elemental range, electron configuration, ring-plus double bond equivalents and comparison of the theoretical with measured isotope patterns was provided in the module. Compounds identified were categorised based on MSI (Metabolomics Standard Initiative) (Sumner et al., 2007).

\section{RESULTS AND DISCUSSION}

One of the challenges in LC-MS metabolomics studies is data analysis and metabolite annotation, i.e. identifying biological molecules from mass spectral data. The highly sensitive LC-MS platform allows the simultaneous detection of multiple analytes combined with data processing workflow using ProfileAnalysis analysis including peak picking, filtering, normalising and statistic analysing (Mamat et al., 2018; Veeramohan et al., 2018; Mazlan et al., 2018; Tahir et al., 2016). In this study, the number of peaks detected from $G$. boninense intracellular metabolites were increased over time, until Day 6 and decreased at Day 8 (Figure 1). The metabolites measured over time were further analysed using PCA. 


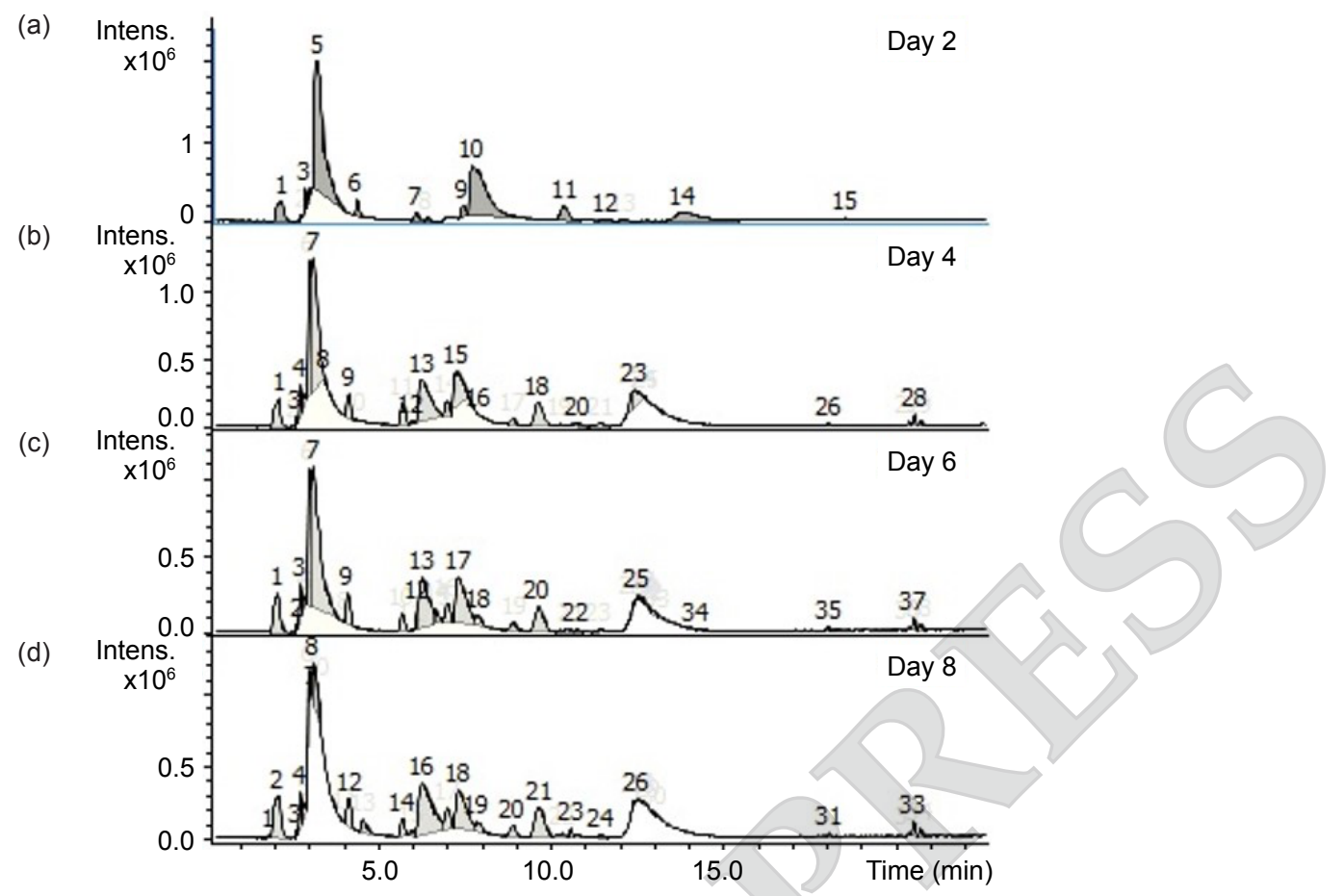

Figure 1. The liquid chromatography-mass spectrometry (LC-MS) base peak chromatograms (BPC) of Ganoderma boninense extracellular metabolites at each time point of Day $2(a), 4(b), 6(c)$ and $8($ d). The highest number of 37 peaks were detected at Day 6 , followed by Day 8 with 33 peaks, Day 4 with 28 peaks and Day 2 with 15 peaks.

\section{Validation of Ganoderma boninense Intracellular Metabolite PCA Model}

PCA was used as a processing tool to enable robust interpretation of large-scale data from the G. boninense intracellular metabolites. Moreover, validation methods are crucial for the evaluation of model performance and prediction ability (Diana and Tommasi, 2002). The rule of cross validation (CV) technique is to test the significance and to correct the confidence limits and was applied to the PCA model for the G. boninense intracellular metabolites. As shown in Figure 2, full cross validation was applied to compare the predicted values with the actual ones. The influence plot of a PCA model for the G. boninense intracellular metabolites showed similar results before (a) and after (b) validation, where, no outlying behaviour outside the quadrant. This indicated the significance of the model at 95\% confidence interval.

Besides, a test set validation of a PCA model was also applied to check the stability of the current model. Test set validation is suitable for large datasets (>50) (Kos et al., 2003). Figure 3 shows the influence plot of the original G. boninense intracellular metabolites PCA model (Figure 3a) and the new model after validation (Figure $3 b$ ). The new model described the test set analyses well, where the basic appearance of the scores plot did not change. This indicated the initial set of analyses all together resulted in a good and valid model.

\section{Ganoderma boninense Intracellular Metabolite PCA Model}

Large multivariate datasets generated from the LC-MS analysis of $G$. boninense intracellular metabolites require pattern recognition techniques that filter the important information from different time points and detect compounds that contribute to the groupings according to time trend. In this study, therefore, we employed an unsupervised method such as PCA to summarise the multidimensional data as well as to capture most of the variance in the datasets. PCA is a mathematical projection technique designed to extract many variables, displays and ranks the variance in a reduced data matrix (Jolliffe and Cadima, 2016). Furthermore, PCA extracts relevant statistics on the distribution of samples and group characteristics (Lazar et al., 2015).

PCA is one of the most important and powerful methods in chemometrics (Bro and Smilde, 2014). Besides, it can also identify the effects of technical variation in the analysis of metabolic profiles, which is crucial for data quality evaluation in metabolomics studies (Gika et al., 2014; Yin et al., 2013). Following the pre-processing LC-MS data of the G. boninense intracellular metabolites, PCA allowed a descriptive evaluation of the samples distribution in order to assess the data quality and to identify natural groupings, patterns and outliers as explained and described in other reports 
(a)

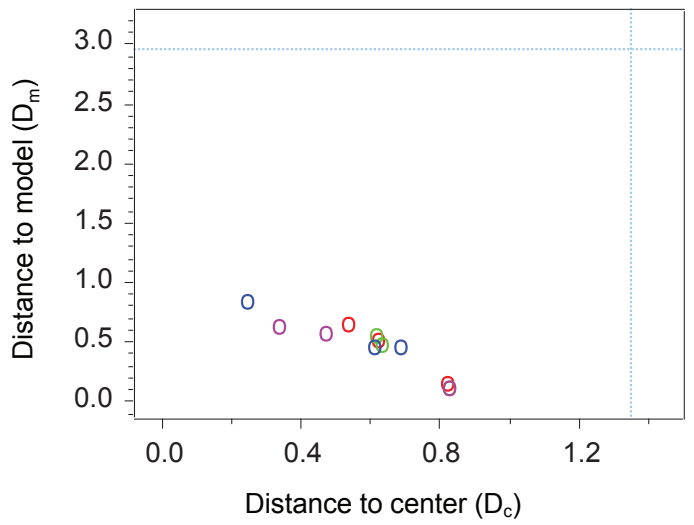

(b)

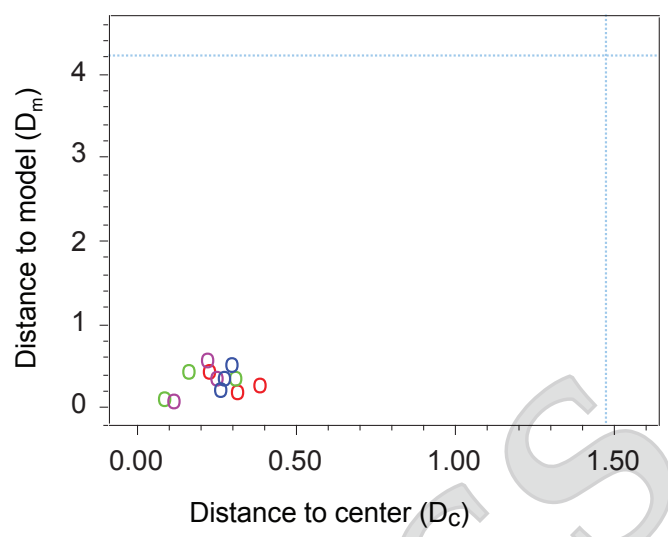

Figure 2. Full cross validation of Ganoderma boninense intracellular metabolite principal component analysis (PCA) model. Influence plots (a) before and (b) after validation show no outlying behaviour outside the quadrant and indicated the significance of the model at 95\% confidence interval.

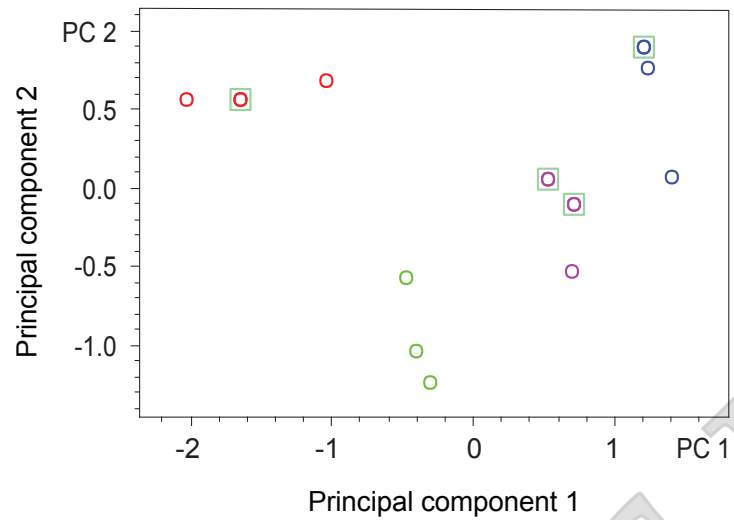

(b)

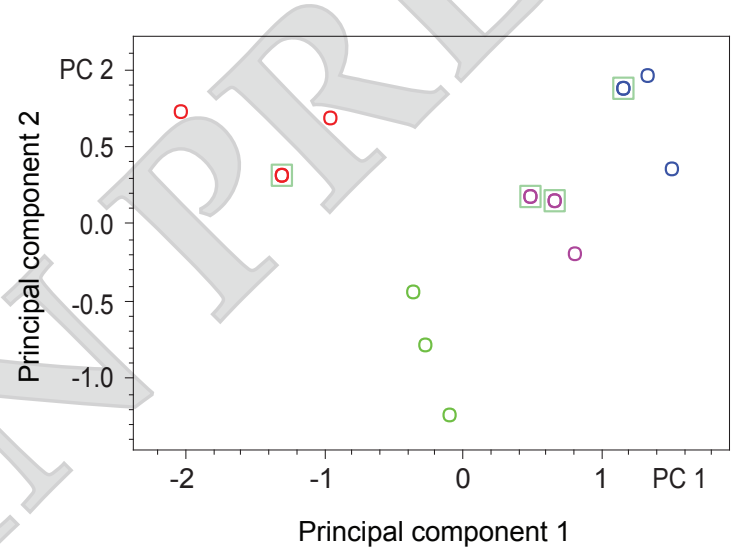

Figure 3. Test validation of Ganoderma boninense intracellular metabolite principal component analysis (PCA) model. The scores plot shows (a) the selection of four test set analyses before $(b)$ and after test set validation. The new PCA model $(b)$ described the test set analyses well, where the basic appearance of the scores plot did not change and resulted in a good and valid model.

(Tugizimana et al., 2019). The samples presented in the PCA scores plot in Figure $4 a$ showed close clustering between data points for each time interval. This indicated a good quality, stability, reliability and reproducibility of the analyses. The scores plot displays the relationship between $G$. boninense intracellular metabolites harvested at the different time intervals of Days 2, 4, 6 and 8 .

Furthermore, in PCA, a linear transformation of the metabolic features in specific directions is called principal components, where they are taken seriously in explaining the most variation of the first component, followed by the second component, etc. (Jolliffe and Cadima, 2016; Alonso et al., 2015). In this study, as shown in the scores plot, PC1 and PC2 explained $87.6 \%$ of the total variance present in the dataset. The first principal component summarises the global dataset since it captures most of the dataset variability in feature/metabolite patterns based on their similarity (Alonso et al., 2015). In our results, PCA models revealed distinct time trend groupings in the G. boninense intracellular metabolites. Here, clear sample groupings indicate dynamic changes occurring in G. boninense developmental process in vitro, and observed as time-dependent stages, related by the time trend which was revealed by PC analyses (Figure $4 b$ ). PCA, as a standard model to deal with high-dimensional and complex metabolomics data, proved to uncover time-trends in a dataset (Tugizimana et al., 2019). The PCA loadings plot (Figure 4b) represents 375 buckets (time and $\mathrm{m} / \mathrm{z}$ ) included in the PCA model. In this model, the four buckets that are far away from the central cloud are responsible for the variance within the dataset; (1) rT $3.6 \mathrm{~min}, \mathrm{~m} / \mathrm{z} 179.0560$, (2) rT 4.5 $\mathrm{min}, \mathrm{m} / \mathrm{z} 195.0514$, (3) rT $2.9 \mathrm{~min}, \mathrm{~m} / \mathrm{z} 181.0719$ and (4) rT $6.6 \mathrm{~min}, \mathrm{~m} / \mathrm{z} 133.0144$.

\section{Ganoderma boninense Intracellular Metabolite Signatures}

From the PCA loadings plot analysis, four metabolites that were located far away from the central cloud, which were responsible for the variance within the dataset were selected and identified as differentially expressed metabolites. 
(a)

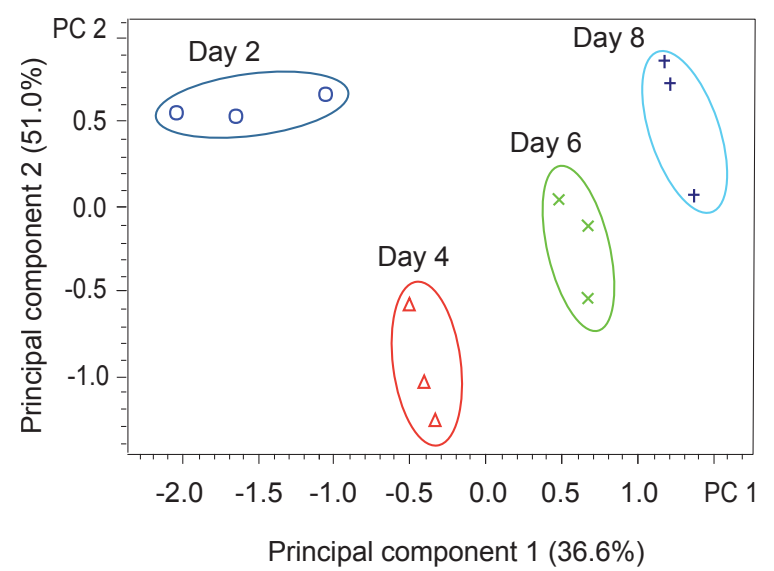

(b)

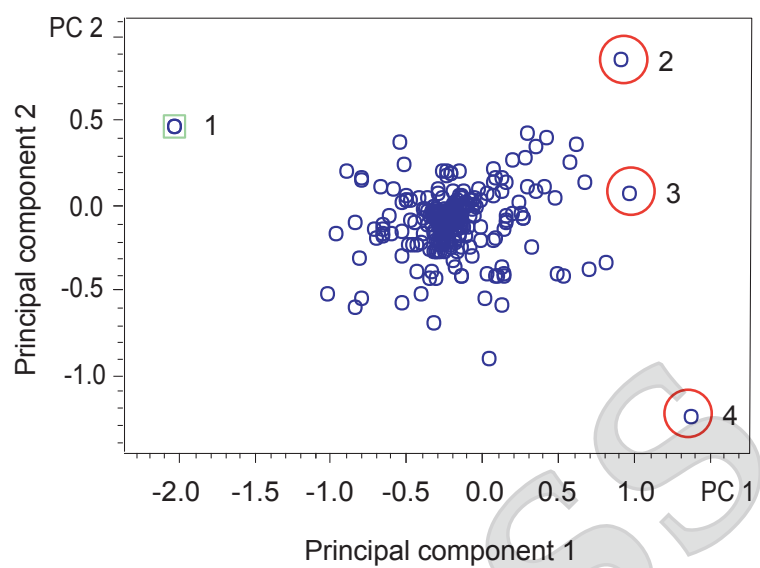

Figure 4. Principal component analysis (PCA) of the Ganoderma boninense intracellular metabolite LC-MS data. Scores plot (a) and loadings plot (b) of negative mode data (Pareto-scaled) from intracellular samples. Each data point in the PCA scores plot represents an analysis included in the PCA model. A 2-component model, explaining $87.6 \%$ of the total variation in the Pareto-scaled data at $95 \%$ confidence level, from each time point at Days 2, 4, 6 and 8. A clear time-related trend is observed.

Metabolites were assigned as (1) glucose $(\mathrm{m} / \mathrm{z}$ 179.0560), (2) gluconic acid ( $m / z$ 195.0514), (3) mannitol $(\mathrm{m} / \mathrm{z}$ 181.0719) and (4) malic acid $(\mathrm{m} / \mathrm{z}$ 133.0144) based on SmartFormula (Bruker Daltonik, Germany), comparison against KEGG database and tandem MS (MS/MS) (Table 1). MS/MS spectra and chemical structures of all identified metabolites were presented on Figures 5-8. According to the PCA loadings plot, glucose showed relatively high abundance at Day 2 while gluconic acid, mannitol and malic acid showed relatively high abundance at Days 4, 6 and 8 , respectively. Bucket statistics plot in the ProfileAnalysis enables a quick visual comparison of the differentially expressed metabolites according to time trend (Figure 9).
The differentially expressed intracellular metabolites in G. boninense, which were classified as primary metabolites, i.e. sugar and organic acid, play significant and essential roles in the growth, reproduction and development of the fungus. These metabolites are associated with the rapid initial growth phase and show maximum production at the end of the phase (Andersen, 2014; Wisecaver et al., 2014; Sanchez and Demain, 2008). Mannitol, for example, is the most abundant soluble carbohydrates and plays a key role as carbon storage/ carbohydrate reserve within the mycelium of fungi (Patel and Williamson, 2016; Wiemken, 2007; Lewis and Smith, 1967). However, mannitol has recently been hypothesised to have a major role in fungal pathogenicity (Patel and Williamson, 2016).

TABLE 1. PROPOSED DIFFERENTIALLY EXPRESSED INTRACELLULAR METABOLITES OF Ganoderma boninense AT DAYS 2, 4, 6 AND 8

\begin{tabular}{|c|c|c|c|c|c|c|c|c|c|c|}
\hline No. & $\begin{array}{c}\mathrm{rT} \\
(\mathrm{min})\end{array}$ & Metabolite & KEGG ID & MSI & $\begin{array}{c}\text { Molecular } \\
\text { formula }\end{array}$ & $\begin{array}{c}\text { Monoisotopic } \\
\text { mass } \\
\left(\mathrm{g} \mathrm{mol}^{-1}\right)\end{array}$ & $\begin{array}{c}m / z \\
\text { measured }\end{array}$ & $\begin{array}{c}m / z \\
\text { theoretical }\end{array}$ & $\begin{array}{l}\text { Error } \\
(\mathrm{ppm})\end{array}$ & $\begin{array}{c}M^{2} \\
\text { fragments }\end{array}$ \\
\hline 1. & 3.6 & Glucose & C00031 & 1 & $\mathrm{C}_{6} \mathrm{H}_{12} \mathrm{O}_{6}$ & 180.06 & 179.0560 & 179.0561 & 0.7 & $\begin{array}{l}161.0433 \\
131.0372 \\
119.0374 \\
101.0252\end{array}$ \\
\hline 2. & 4.5 & $\begin{array}{l}\text { Gluconic } \\
\text { acid }\end{array}$ & C00257 & 2 & $\mathrm{C}_{6} \mathrm{H}_{12} \mathrm{O}_{7}$ & 196.06 & 195.0514 & 195.0510 & -2.0 & $\begin{array}{l}177.0398 \\
159.0282 \\
129.0180\end{array}$ \\
\hline 3. & 2.9 & Mannitol & C00392 & 2 & $\mathrm{C}_{6} \mathrm{H}_{14} \mathrm{O}_{6}$ & 182.08 & 181.0719 & 181.0718 & -0.6 & $\begin{array}{l}161.0465 \\
119.0334 \\
113.0217 \\
101.0222\end{array}$ \\
\hline 4. & 6.6 & Malic acid & C00711 & 2 & $\mathrm{C}_{4} \mathrm{H}_{6} \mathrm{O}_{5}$ & 134.02 & 133.0144 & 133.0142 & -1.4 & $\begin{array}{c}115.0017 \\
89.0189\end{array}$ \\
\hline
\end{tabular}


(a)

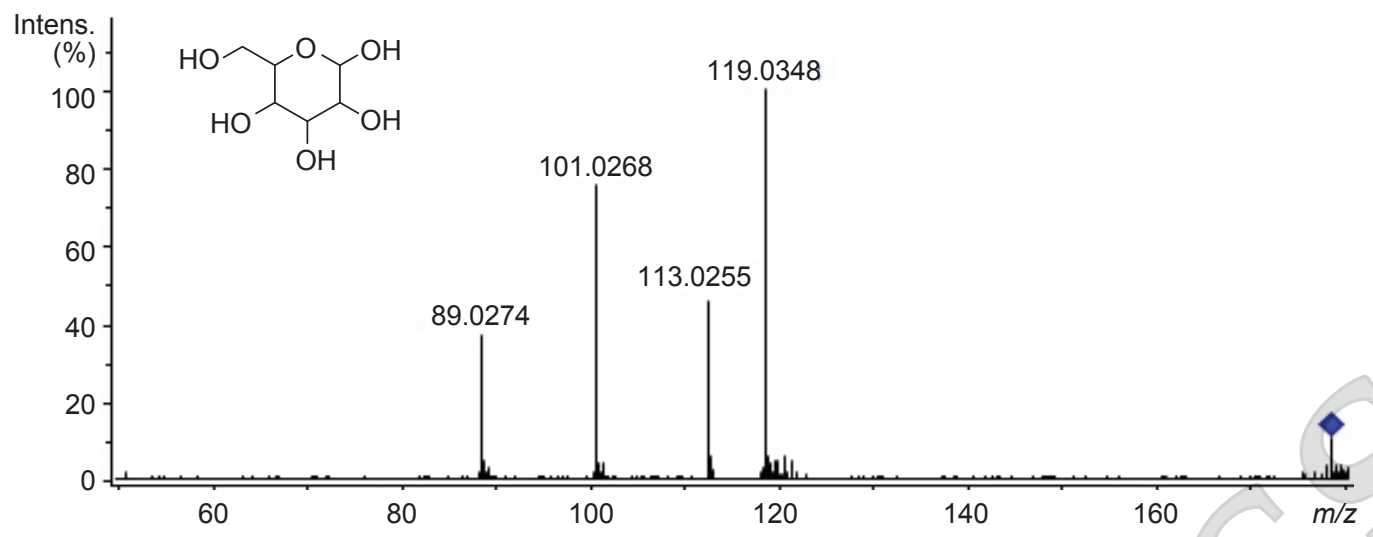

(b)

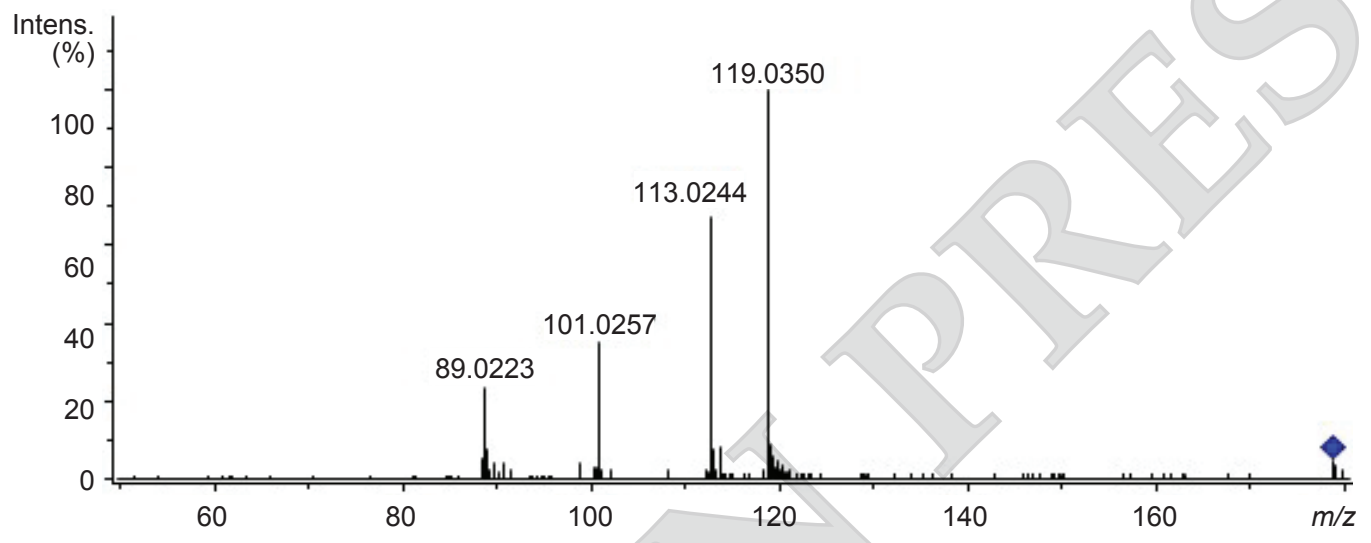

Figure 5. Negative ion mode mass spectrometry/mass spectrometry (MS/MS) spectra of glucose (m/z 179.0560, $\left.\mathrm{C}_{6} \mathrm{H}_{12} \mathrm{O}_{6}\right)$ at a collision energy of $20 \mathrm{eV}$. Similar MS/MS spectra of (a) glucose in Ganoderma boninense mycelia sample and (b) glucose reference standard.

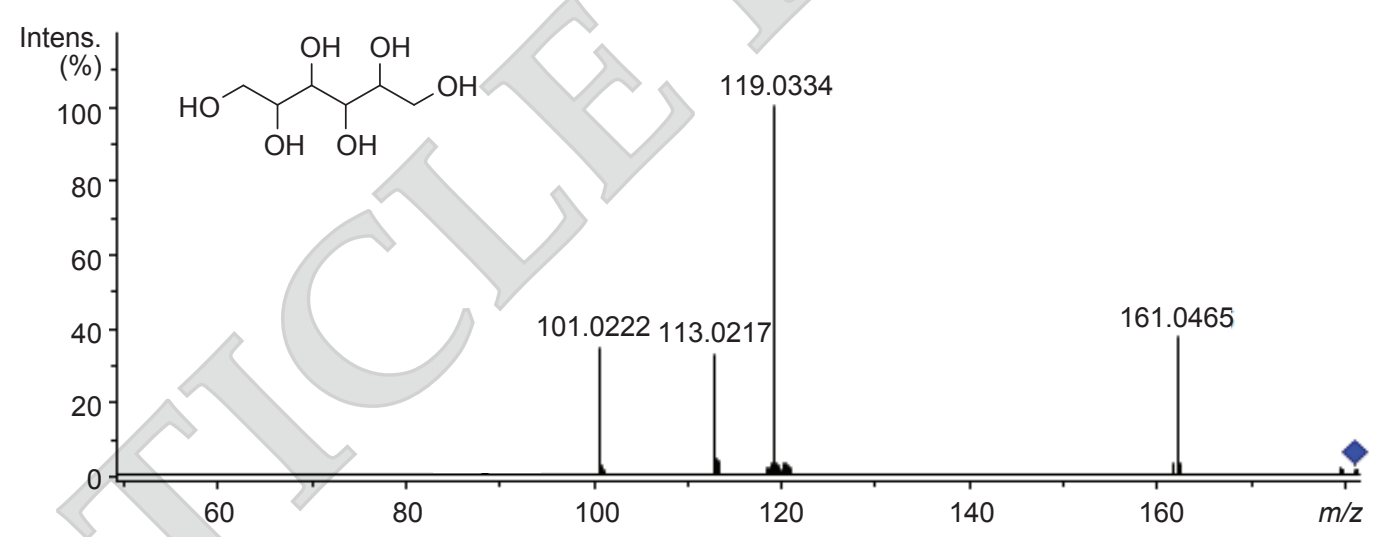

Figure 6. Negative ion mode mass spectrometry/mass spectrometry (MS/MS) spectrum of mannitol (m/z 181.0719, $\left.\mathrm{C}_{6} \mathrm{H}_{14} \mathrm{O}_{6}\right)$ at a collision energy of $35 \mathrm{eV}$.

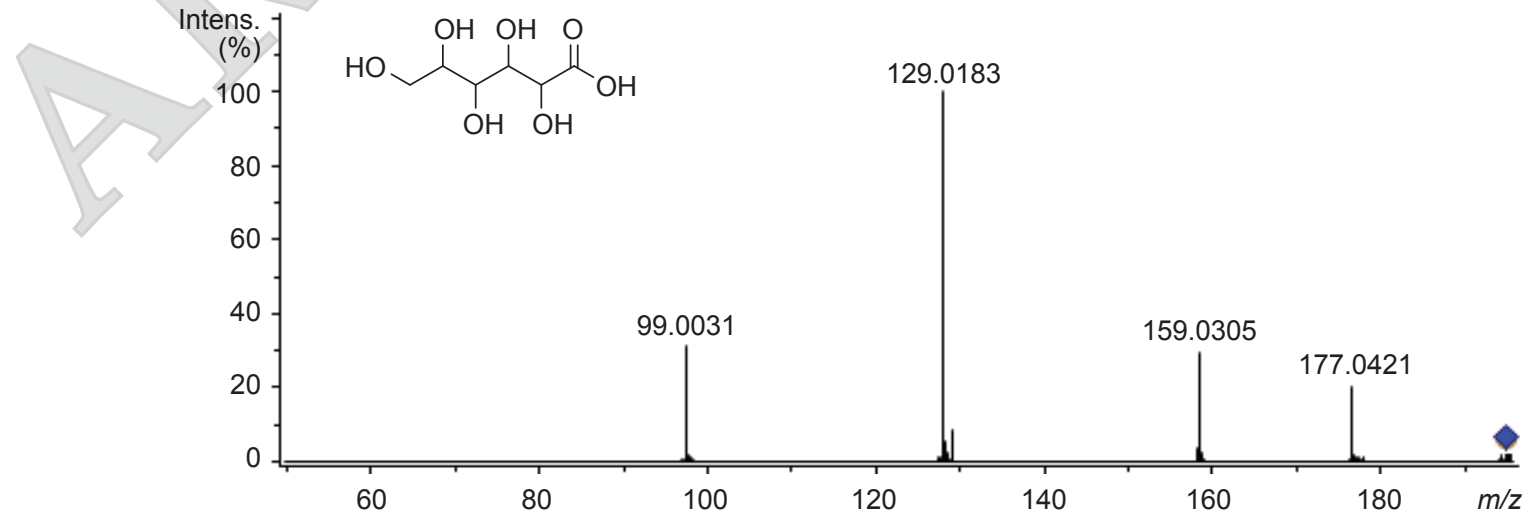

Figure 7. Negative ion mode mass spectrometry/mass spectrometry (MS/MS) spectra of gluconic acid (m/z 195.0514, $\left.\mathrm{C}_{6} \mathrm{H}_{12} \mathrm{O}_{7}\right)$ at a collision energy of $15 \mathrm{eV}$. 


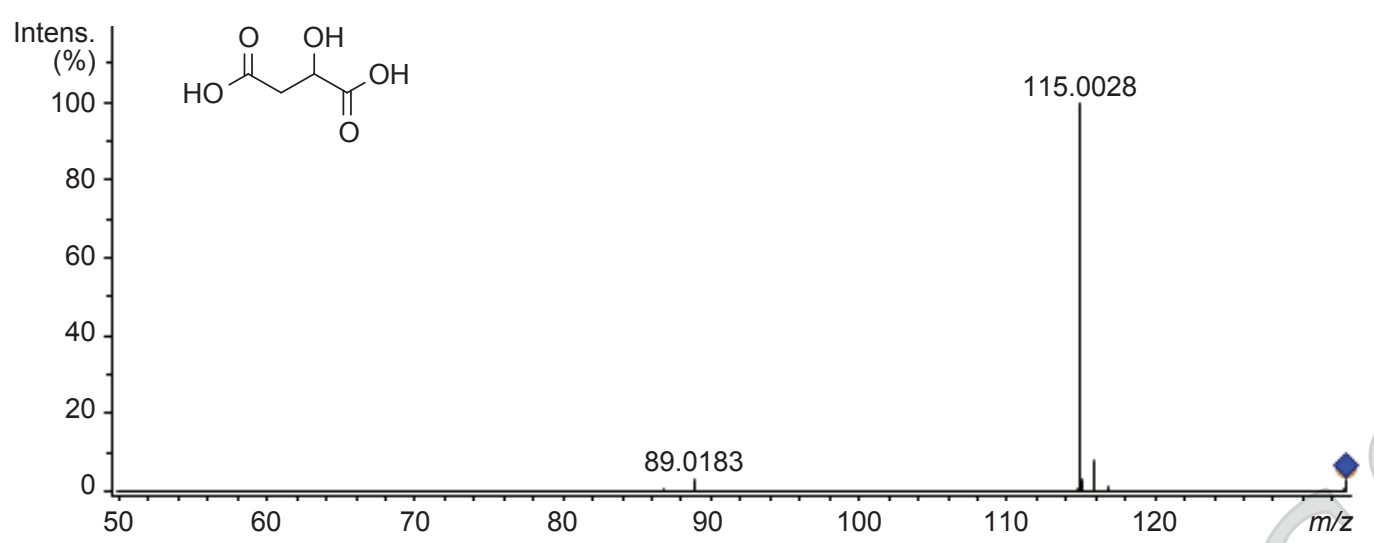

Figure 8. Negative ion mode mass spectrometry/mass spectrometry (MS/MS) spectra of malic acid ( $\mathrm{m} / \mathrm{z} 133.0144, \mathrm{C}_{4} \mathrm{H}_{6} \mathrm{O}_{5}$ ) at a collision energy of $15 \mathrm{eV}$.

(a)

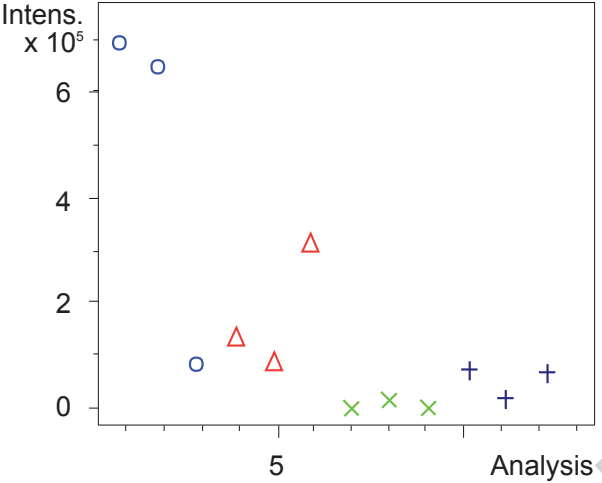

(c)

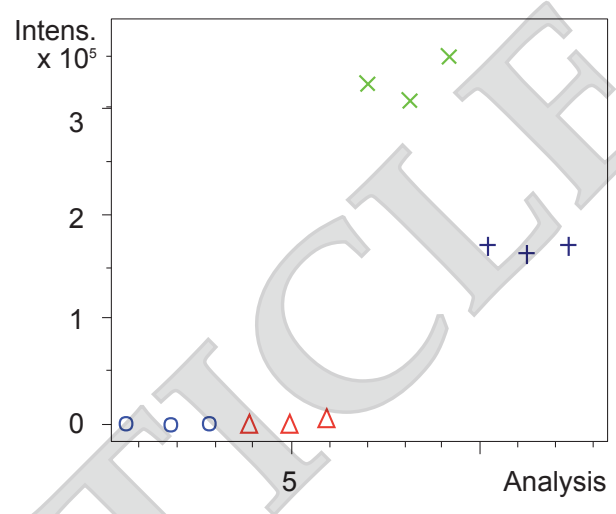

(b)

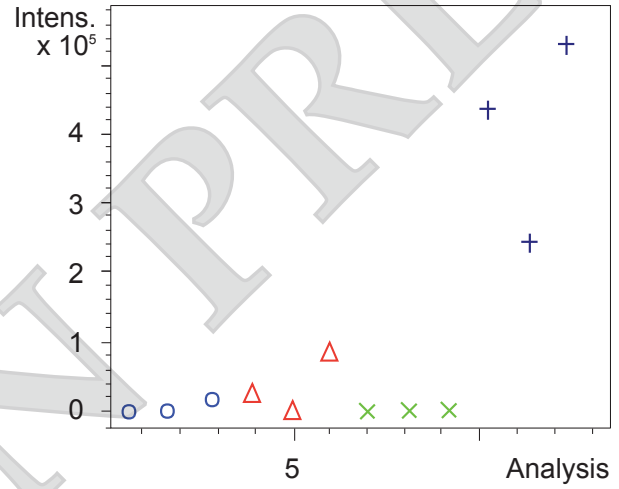

(d)

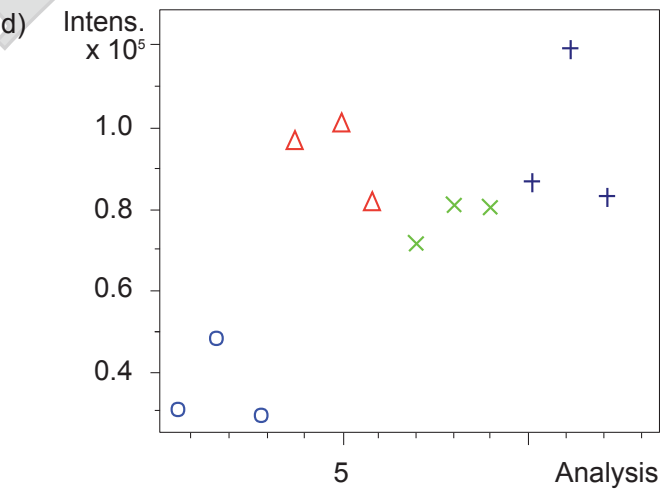

Figure 9. Bucket statistic plot of four differential metabolites observed in the loadings plot of principal component analysis $(P C A)(\bullet$ for Day $2, \triangle$ for Day 4, $\times$ for Day 6 and + for Day 8). (a) Glucose $\left(\mathrm{C}_{6} \mathrm{H}_{12} \mathrm{O}_{6}, \mathrm{~m} / \mathrm{z}\right.$ 179.0560), (b) gluconic acid $\left(\mathrm{C}_{6} \mathrm{H}_{12} \mathrm{O}_{7}, \mathrm{~m} / \mathrm{z}\right.$ 195.0514), (c) mannitol $\left(\mathrm{C}_{6} \mathrm{H}_{14} \mathrm{O}_{6}\right.$, $\mathrm{m} / \mathrm{z} 181.0719)$ and (d) malic acid $\left(\mathrm{C}_{4} \mathrm{H}_{6} \mathrm{O}_{5}, \mathrm{~m} / \mathrm{z} 133.0144\right)$.

\section{CONCLUSION}

Ganoderma boninense is an economically important pathogen in oil palm where a holistic and detailed understanding of the pathogen biology may significantly improve targeted disease control and management strategies. The present study provides a necessary step for uncovering molecular events during the developmental process of G. boninense using LC-MS and multivariate statistical data analysis, particularly PCA. Albeit the interpretation of the large and complex
LC-MS datasets of fungal metabolites is frequently challenging, our studies have demonstrated that PCA provides a robust and reliable comparison of temporal intracellular metabolite changes of $G$. boninense. This may indicate the dynamic changes occurring in G. boninense developmental process, in vitro. Furthermore, this information is of key importance that can complement the available transcriptomics and proteomics data of G. boninense, towards further understanding the $G$. boninense pathogenicity. 


\section{ACKNOWLEDGEMENT}

The authors would like to thank the DirectorGeneral of MPOB for permission to publish this article. We would also like to thank the members of the Proteomics and Metabolomics Unit, Advanced Biotechnology and Breeding Centre (ABBC), MPOB for their technical support and invaluable contribution. We acknowledge the support and assistance of Jayanthi Nagappan, Bioinformatics Unit, ABBC.

\section{REFERENCES}

Alonso, A; Marsal, S and Julia, A (2015). Analytical methods in untargeted metabolomics: State of the art in 2015. Front. Bioeng. Biotechnol., 3: 23-23.

Andersen, M R (2014). Elucidation of primary metabolic pathways in Aspergillus species: Orphaned research in characterizing orphan genes. Brief Funct. Genomics, 13: 451-455.

Bro, R and Smilde, A K (2014). Principal component analysis. Anal. Methods, 6: 2812-2831.

Chen, F; Ma, R and Chen, X L (2019). Advances of metabolomics in fungal pathogen-plant interactions. Metabolites, 9(8): 169.

Chong, K P; Dayou, J and Alexander, A (2017). Pathogenic nature of Ganoderma boninense and basal stem rot disease. Detection and Control of Ganoderma boninense in Oil Palm Crop. Springer. p. 5-12.

De Falco, B; Manzo, D; Incerti, G; Garonna, A P; Ercolano, M and Lanzotti, V (2019). Metabolomics approach based on NMR spectroscopy and multivariate data analysis to explore the interaction between the leafminer Tuta absoluta and tomato (Solanum lycopersicum). Phytochem. Anal., 30: 556-563.

DesRochers, N; Walsh, J P; Renaud, J B; Seifert, K A; Yeung, K K-C and Sumarah, M W (2020). Metabolomic profiling of fungal pathogens responsible for root rot in American ginseng. Metabolites, 10: 35.

Diana, G and Tommasi, C (2002). Cross-validation methods in principal component analysis: A comparison. Stat. Methods Appl., 11: 71-82.

Gika, H G; Theodoridis, G A; Plumb, R S and Wilson, I D (2014). Current practice of liquid chromatography-mass spectrometry in metabolomics and metabonomics. J. Pharm. Biomed. Anal., 87: 12-25.
Gotthardt, M; Kanawati, B; Schmidt, F; Asam, S; Hammerl, R; Frank, O; Hofmann, T; SchmittKopplin, P and Rychlik, M (2019). Comprehensive analysis of the Alternaria mycobolome using mass spectrometry based metabolomics. Mol. Nutr. Food Res., 64(3): 1900558.

Hayden, H L; Rochfort, S J; Ezernieks, V; Savin, K W and Mele, P M (2019). Metabolomics approaches for the discrimination of disease suppressive soils for Rhizoctonia solani AG8 in cereal crops using 1H NMR and LC-MS. Sci. Total. Environ., 651(1):16271638.

Holmes, E and Antti, H (2002). Chemometric contributions to the evolution of metabonomics: Mathematical solutions to characterising and interpreting complex biological NMR spectra. Analyst, 127: 1549-1557.

Ivosev, G; Burton, L and Bonner, R (2008). Dimensionality reduction and visualization in principal component analysis. Anal. Chem., 80: 49334944.

Jolliffe, IT and Cadima, J (2016). Principal component analysis: A review and recent developments. Philos. Trans. A. Math. Phys. Eng. Sci., 374: 20150202.

Jonsson, P; Bruce, S J; Moritz, T; Trygg, J; Sjöström, M; Plumb, R; Granger, J; Maibaum, E; Nicholson, J K and Holmes, E (2005). Extraction, interpretation and validation of information for comparing samples in metabolic LC/MS data sets. Analyst, 130: 701-707.

Kos, G; Lohninger, H and Krska, R (2003). Validation of chemometric models for the determination of deoxynivalenol on maize by mid-infrared spectroscopy. Mycotoxin Res., 19: 149-153.

Lazar, A G; Romanciuc, F; Socaciu, M A and Socaciu, C (2015). Bioinformatics tools for metabolomic data processing and analysis using untargeted liquid chromatography coupled with mass spectrometry. Bull. Univ. Agric. Sci., 72: 103-115.

Lewis, D and Smith, D (1967). Sugar alcohols (polyols) in fungi and green plants. I. Distribution, physiology and metabolism. New Phytol., 66: 143-184.

Liu, H; Zhao, X; Guo, M; Liu, H and Zheng, Z (2015). Growth and metabolism of Beauveria bassiana spores and mycelia. BMC Microbiol., 15: 267 pp.

Mamat, S F; Azizan, K A; Baharum, S N; Noor, N M and Aizat, W M (2018). ESI-LC-MS basedmetabolomics data of mangosteen (Garcinia mangostana Linn.) fruit pericarp, aril and seed at different ripening stages. Data Brief, 17: 1074-1077. 
Mazlan, O; Aizat, W M; Aziz Zuddin, N S; Baharum, S N and Noor, N M (2018). LC-MS data for metabolomics analysis of Garcinia mangostana L. seed germination. Data Brief, 21: 2221-2223.

Othman, A; Abd Rasid, O; Nagappan, J; Leslie Low, E T; Fook Hwa, L; Nurazah, Z; Syahanim, S; Dzulkafli, S B; Rozali, N L; Bohari, B; Angel, L P L; Tahir, N I; Idris, A S; Marjuni, M; Sundram, S; Mohd Din, A; Ramli, U S and Mohamad Arif, A M (2019). Molecular characterisation of oil palm responses to Ganoderma infection. International Seminar on Breeding for Ganoderma Tolerance in Oil Palm. Kuala Lumpur Convention Centre, Kuala Lumpur. p. 137-146.

Patel, T K and Williamson, J D (2016). Mannitol in plants, fungi, and plant-fungal interactions. Trends Plant Sci., 21: 486-497.

Rees, R W (2006). Ganoderma stem rot of oil palm (Elaeis guineensis): Mode of infection, epidemiology and biological control. Ph.D thesis, University of Bath, United Kingdom.

Robison, F M; Turner, M F; Jahn, C E; Schwartz, H F; Prenni, J E; Brick, M A and Heuberger, A L (2018). Common bean varieties demonstrate differential physiological and metabolic responses to the pathogenic fungus Sclerotinia sclerotiorum. Plant Cell Environ., 41: 2141-2154.

Sanchez, S and Demain, A L (2008). Metabolic regulation and overproduction of primary metabolites. Microb. Biotechnol., 1: 283-319.

Siless, G E; Gallardo, G L; Rodriguez, M A; Rincon, Y A; Godeas, AM and Cabrera, GM (2018). Metabolites from the dark septate endophyte Drechslera sp. evaluation by LC/MS and principal component analysis of culture extracts with histone deacetylase inhibitors. Chem. Biodivers., 15: e1800133.

Son, S Y; Lee, S; Singh, D; Lee, N-R; Lee, D-Y and Lee, C H (2018). Comprehensive secondary metabolite profiling toward delineating the solid and submerged-state fermentation of Aspergillus oryzae KCCM 12698. Front. Microbiol., 9: 1076-1076.

Sumner, L W; Amberg, A; Barrett, D; Beale, M H; Beger, R; Daykin, C A; Fan, T W-M; Fiehn, O; Goodacre, R and Griffin, J L (2007). Proposed minimum reporting standards for chemical analysis. Metabolomics, 3: 211-221.

Tahir, N I; Shaari, K; Abas, F; Ishak, Z and Tarmizi, A (2016). Metabolome analysis of oil palm clone P325 of different planting trials. J. Oil Palm Res. Vol. 28: 431-441.

Tugizimana, F; Djami-Tchatchou, A T; Fahrmann, J F; Steenkamp, P A; Piater, LA and Dubery, I A (2019). Time-resolved decoding of metabolic signatures of in vitro growth of the hemibiotrophic pathogen Colletotrichum sublineolum. Sci. Rep., 9: 3290.

Veeramohan, R; Azizan, K A; Aizat, W M; Goh, H-H; Mansor, S M; Yusof, N S M; Baharum, S N and $\mathrm{Ng}, \mathrm{C} \mathrm{L}$ (2018). Metabolomics data of Mitragyna speciosa leaf using LC-ESI-TOF-MS. Data Brief, 18: 1212-1216.

Wiemken, V (2007). Trehalose synthesis in ectomycorrhizas-a driving force of carbon gain for fungi? New Phytol., 174: 228-230.

Wisecaver, J H; Slot, J C and Rokas, A (2014). The evolution of fungal metabolic pathways. PLoS Genet., 10: e1004816.

Yin, P; Peter, A; Franken, H; Zhao, X; Neukamm, S S; Rosenbaum, L; Lucio, M; Zell, A; Haring, H U; Xu, G and Lehmann, R (2013). Preanalytical aspects and sample quality assessment in metabolomics studies of human blood. Clin. Chem., 59: 833-845.

Yuan, S; Yan, J; Wang, M; Ding, X; Zhang, Y; Li, W; Cao, J and Jiang, W (2019). Transcriptomic and metabolic profiling reveals 'Green Ring' and 'Red Ring' on jujube fruit upon postharvest Alternaria alternata infection. Plant Cell Physiol., 60: 844-861. 